\title{
Descriptive audit of maternal sepsis in a tertiary care centre of North India
}

\section{Archana Kumari, Jyotsna Suri*, Pratima Mittal}

Department of Obstetrics and Gynecology, VMMC and Safdarjung Hospital, New Delhi, India

Received: 28 November 2017

Accepted: 04 December 2017

\section{*Correspondence:}

Dr. Jyotsna Suri,

E-mail: jyotsnasuri@gmail.com

Copyright: (C) the author(s), publisher and licensee Medip Academy. This is an open-access article distributed under the terms of the Creative Commons Attribution Non-Commercial License, which permits unrestricted non-commercial use, distribution, and reproduction in any medium, provided the original work is properly cited.

\begin{abstract}
Background: Maternal sepsis is one of the leading causes of maternal mortality around the world. The aim of this study was to study the prevalence, clinical profile and fetomaternal outcome of maternal sepsis at a dedicated Obstetric critical care unit of a tertiary care centre of North India. It was retrospective study conducted in tertiary care centre in North India

Methods: Women diagnosed as sepsis or septic shock at any point in pregnancy and up to 6 weeks postpartum (irrespective of the source of infection) were included in the study. Demographic, clinical, microbiological and outcome data were recorded from the case sheets of all patients admitted in obstetrical critical care unit between January to December 2016. Outcome measures: Prevalence, bacterial organism, source of infection, mode of delivery, period of gestation, maternal and fetal outcome.

Results: The prevalence of maternal sepsis was 16.5/10,000 live births. The number of maternal deaths attributable to sepsis were 35 , making the maternal mortality ratio due to sepsis $128 / 100,000$ live births. $87 \%$ of the cases were unbooked. $22 \%$ presented antenatally while $58 \%$ were postpartum and $20 \%$ were postabortal. Genital tract infection was most common source of infection. E. coli was the predominant organism in $28 \%$ followed by Methicillin resistant staphylococcus aureus in $12 \%$. The mortality from sepsis was very high (78\%). 54\% of cases required mechanical ventilation and around $7 \%$ had to be shifted to intensive care unit for advanced life support and care. Sepsis was associated with preterm delivery and a high perinatal mortality rate.

Conclusions: Early recognition of the severity of infection and prompt management by a multidisciplinary team of intensivists, anesthetists, neonatologists, obstetrician, midwives are the key to success. Vigilant infection control measures must be strictly practiced during all pregnancy events.
\end{abstract}

Keywords: Fetomaternal outcome, Maternal mortality, Maternal sepsis

\section{INTRODUCTION}

Maternal sepsis is a leading cause of preventable maternal morbidity and mortality in our country. Mostly, it is due to peripartum factors and the instigating organisms are usually from the polymicrobial flora of the genitourinary tract. ${ }^{1}$ However, non-obstetric infections that may get aggravated due to physiological alterations in pregnancy, also make a significant contribution to morbidity due to infectious etiologies in pregnancy.

The majority of the cases of sepsis present within 24-48 hours of delivery, abortion, or rupture of membranes. ${ }^{2}$ The expeditious recognition of maternal sepsis and meticulous and appropriate management can prevent the progression to severe sepsis and septic shock. 
Studies from developed countries report maternal mortality ratio from sepsis to be $1.13 / 100,000 .^{3}$ In these countries, maternal sepsis is usually the result of puerperal sepsis and urinary tract infections. ${ }^{4}$

Little is known about the epidemiology, etiology, incidence of severe maternal morbidity related to sepsis and outcome of maternal sepsis in low-income countries like India. A significant number of maternal deaths are expected to be associated with risk factors due to poverty such as delivery without a trained birth assistant, lack of accessibility to healthcare facilities etc. ${ }^{5,6}$

The overall risk of mortality and morbidity from maternal sepsis can be reduced by improving maternal and child care services. In view of the increasing rates and severity of sepsis worldwide and limited data from developing countries like ours, we planned this study to find out the clinical profile and fetomaternal outcome of maternal sepsis at a dedicated obstetric critical care unit of a tertiary care centre in India.

\section{METHODS}

This retrospective observational study was done at Department of Obstetrics and Gynecology, VMMC and Safdarjung Hospital, New Delhi. VMMC and Safdarjung Hospital is a tertiary care hospital located in the capital of India. More than 25,000 deliveries occur annually, which is highest in any single hospital in India. Recently, a dedicated obstetrical critical care unit with state of art ventilators and modern equipments was started here and it is the first of its kind in any government hospital in Northern India. Case record sheets of all patients admitted in obstetrical critical care unit between January 2016 to December 2016 were studied and findings were recorded in a predesigned performa. The study was approved by Institutional ethics committee.

Pregnant women diagnosed as sepsis or septic shock at any point in pregnancy and up to 6 weeks postpartum (irrespective of the source of infection) were included in the study. The clinical diagnosis of sepsis was made when following occurred at two occasions at least 4 hours apart: temperature $>38^{\circ} \mathrm{C}$ or $<36^{\circ} \mathrm{C}$, Heart rate $\geq 100$ beats/min, respiratory rate $\geq 20 / \mathrm{min}, \mathrm{WBC}>17 \times 109 / 1$ or $<4 \times 109 / 1$ or $>10 \%$ immature BF. Besides, patients who died due to infection or suspected infection or required level 2 or level 3 critical care with suspected severe sepsis were also included.

The clinico-demographic, microbiological and outcome data were recorded from the patient's case sheet. Obstetric information recorded included parity, booking status, gestation or postpartum day at time of sepsis, mode of delivery and gestation at that time, place of delivery, history of prior antibiotic treatment. Outcome data recorded included maternal admission to an Intensive care unit, gestation at time of delivery, mode of delivery, infectious complications and maternal, fetal or early neonatal death. Evidence of fetal infection in cases of miscarriage, stillbirth, early neonatal death and preterm birth was determined based on clinical, microbiological and histological findings.

\section{RESULTS}

\section{General considerations}

During the study period, 719 patients were admitted in the obstetrical critical care unit, of which 45 patients had fulfilled the criteria of sepsis and were included in the study. General characteristics of the patients included in the study are given in Table 1 .

Table 1: General characteristics of the patients admitted with sepsis in obstetrical critical care unit.

\begin{tabular}{|l|l|}
\hline Characteristics of the patients & Value \\
\hline Age of the patient (in years) & \\
\hline Less than 20 years & $31 \%$ \\
\hline $20-35$ years & $09 \%$ \\
\hline More than 35 years & $60 \%$ \\
\hline Parity & \\
\hline 1 & $40 \%$ \\
\hline 2 & $33 \%$ \\
\hline 3 or more & $27 \%$ \\
\hline Booking status & \\
\hline Registered & $11 \%$ \\
\hline Booked & $02 \%$ \\
\hline Unbooked & $87 \%$ \\
\hline Antecedent pregnancy event & \\
\hline Antenatal & $22 \%$ \\
\hline Postnatal & $58 \%$ \\
\hline Postabortal & $20 \%$ \\
\hline Place of delivery & \\
\hline Home & $20 \%$ \\
\hline Outside & $51 \%$ \\
\hline In hospital & $19 \%$ \\
\hline History of prior antibiotic treatment & $66 \%$ \\
\hline Yes & $34 \%$ \\
\hline No & \\
\hline
\end{tabular}

The total number of live births over the study period was 27,202. The prevalence of maternal sepsis was $16.5 / 10,000$ live births. The number of maternal deaths attributable to sepsis was 35 , making the maternal mortality ratio of $128 / 100,000$ live births.

Of the 45 cases of maternal sepsis, 26 were postnatal women. Among them, 12 had a normal vaginal delivery while 14 had a cesarean section. All except 2 had an emergency cesarean section. $20 \%$ of the patients of maternal sepsis were post-abortal and all of them were induced abortions and referred by other hospitals/ maternity centers. $20 \%$ of the patients had history of home delivery while $51 \%$ had delivered at maternity centers other than our hospital. All the patients who had been referred to our centre had already received antibiotic 
treatment. $35 \%$ had not received any antibiotics till the time of admission in obstetrical care unit. $60 \%$ of the cases presented within 7 days of delivery while $11 \%$ presented after $10^{\text {th }}$ postpartum day.

\section{Risk factors}

Age emerged as an important risk factor for maternal sepsis as $60 \%$ of patients belonged to age group more than 35 years. Booking status of the mother was also important risk factor as approximately $90 \%$ of the cases of sepsis were unbooked at the time of presentation.

\section{Spectrum of Infection and causative organism}

Genital tract infection was present in $51 \%$ of the women with sepsis. This was followed by chest infection (25\%), wound infection (7\%), urinary tract infection (5\%) while in $6 \%$ of the patients, the site of infection remained undiagnosed.

E. coli was identified in $28 \%$ of the cases followed by MRSA in $12 \%$, streptococcus in $7 \%$ of the cases. No microorganisms could be isolated in $40 \%$ of the patients.

\section{Outcome}

The mortality from sepsis was very high $(80 \%$ which is 35 out of 45 cases). $54 \%$ of cases required mechanical ventilation and around $7 \%$ were shifted from the critical care unit to intensive care unit for advanced life support and care. The duration of hospital stay was less than 48 hours in $42 \%$ of the cases. One out of ten cases stayed for more than 7 days.

An obstetrical procedure had to be performed in $13 \%$ of the cases. Repeat laparotomy was required in 3 out of 45 patients for pyoperitoneum drainage with bowel repair while hysterectomy was done for secondary postpartum hemorrhage in 1 patient. Uterine curettage was done in two cases for removal of infected retained products of conception.

One-third of the patients had multiorgan failure and while pulmonary edema occurred in $24 \%$ of the patients. $33 \%$ of the cases were in disseminated intravascular coagulation and around $38 \%$ had acute renal failure.

On death review of maternal death due to sepsis, delay in seeking timely help was found in $42 \%$ of the cases while $38 \%$ were referred after significant delay to our tertiary care centre. In $20 \%$ of the cases delay was found in obtaining legal abortion.

\section{DISCUSSION}

Sepsis is a life-threatening condition in which there is organ dysfunction due to infection. Septic shock is a subset of sepsis in which there is profound circulatory, cellular, and metabolic abnormalities. Patients with septic shock can be clinically identified low blood pressure with requirement of vasopressors to maintain a mean arterial pressure of $65 \mathrm{~mm} \mathrm{Hg}$ or greater and serum lactate level greater than $2 \mathrm{mmol} / \mathrm{L}(>18 \mathrm{mg} / \mathrm{dL})$ in the absence of hypovolemia. This combination is associated with hospital mortality rates greater than $40 \% .^{1}$

Sepsis in obstetrical cases can be directly pregnancyrelated or as a condition aggravated by normal pregnancy physiology, which ultimately have the potential to progress to severe sepsis and septic shock. The focus of sepsis can vary according to the stage of pregnancy and includes genital tract sepsis (which may extend from cervicitis and endometritis through to pelvic thrombophlebitis and peritonitis), urosepsis, surgical site infection, pneumonia, empyema and mastitis. ${ }^{2}$

The prevalence of maternal sepsis was estimated to be $16.5 / 10,000$ live births consistent with reported rates of $0.1-0.3 \%$ for developed countries. ${ }^{7-10}$

Sepsis has a very high fatality rate among obstetric patients. Puerperal sepsis is a major cause of maternal mortality $(31.5 \%)$. Unbooked status and unsupervised delivery is an important risk factor for the development of maternal sepsis. Genital tract infection and chest infection are the most common causes of infection. Emergency cesarean section carries a significant risk for the development of sepsis. E. coli and MRSA infection account for the majority of sepsis cases. The finding that maternal sepsis progressed in $60 \%$ of the cases who had already been started on antibiotics highlights the complexity involved in the management of sepsis patients.

The altered maternal physiology due to pregnancy and sepsis does affect the pharmacokinetics and pharmacodynamics of drugs. The standard antibiotics may be inappropriate and inadequate for this population requiring apt knowledge and expertise before prescription in order to prevent antibiotic resistance due to irrational use. Furthermore, simply prescribing antibiotics alone would not serve any purpose unless adequate follow up is ensured to find out the effectiveness of the treatment prescribed.

Delay in referral to tertiary care level was found in $38 \%$ of the patients. This reflects upon the fact that most of the patients initially present to the primary care general practitioners or emergency medical team who may be less aware of the sign and symptoms of sepsis or even the rapidity of progression of sepsis. Most patients presented within 7 days of delivery however they were already in a state of irreversible damage. Hence it is the need of the hour to sensitize the general practitioners and emergency team about maternal sepsis and encourage them to have a low threshold for referral to tertiary care centre without any delay. 
The increased rate of maternal sepsis associated with emergency cesarean section highlights the need for antibiotic prophylaxis within 60 minutes before cesarean section. Furthermore, stringent infection control measures must be followed at all the steps of care of this population.

Results of the present study must be interpreted in the light of several limitations. First, it is a retrospective study and the data collected is too small to generalize the results for larger population. Second, in the absence of data for control groups it is not possible to comment on the temporality of factors like mode of delivery, age, and parity with respect to development or progression of sepsis.

The results of the study have significant implications for health care delivery system in India. In view of the high prevalence of maternal sepsis in our centre and a very high case fatality rate, there is need for development of national guidelines for prevention and management of maternal sepsis in our country. Institutional delivery should be promoted in order to prevent and promptly manage any complication of labour and delivery. Intensive care and monitoring is required for cases of maternal sepsis. Hence it is imperative to train the primary care practitioners to recognize the sign and symptoms of sepsis and have a low threshold for transfer to tertiary care level. At all levels of care, the government should look into the measures to prevent the overcrowding and increase the doctor:patient and nurse:patient ratio to provide quality care..

\section{CONCLUSION}

Maternal sepsis is a preventable cause of maternal morbidity and mortality. Early recognition of the severity of infection and institution of effective maternal resuscitation by a multidisciplinary team of intensivists, anaesthetists, neonatologists, obstetrician, midwives are the key to success.

Primary care practitioners should have a low threshold for referral of women in pregnancy with signs of infection. Improving antenatal care, increasing the number of booked patients, selection of high risk cases for mandatory hospital delivery and timely referrals are some of the steps to improve the outcome. Vigilant infection control measures must be strictly practiced during all pregnancy events.

\section{Funding: No funding sources} Conflict of interest: None declared

Ethical approval: The study was approved by the Institutional Ethics Committee

\section{REFERENCES}

1. Surgers L, Valin N, Carbonne B, Bingen E, Lalande $\mathrm{V}$, Pacanowski J, et al. Evolving microbiological epidemiology and high fetal mortality in 135 cases of bacteremia during pregnancy and postpartum. Eur $\mathbf{J}$ Clin Microbiol Infect Dis. 2013;32:107-13.

2. Ronsmans C, Graham WJ. Lancet maternal survival series steering group. Maternal mortality: who, when, where, and why. Lancet. 2006;368:1189-200.

3. Cantwell R, Clutton-Brock T, Cooper G. Centre for Maternal and Child Enquiries (CMACE). Saving mothers' lives: reviewing maternal deaths to make motherhood safer: 2006-08. The eighth report on confidential enquiries into maternal deaths in the United Kingdom. BJOG. 2011;118:1-203.

4. Maupin RT. Obstetric infection disease emergencies. Clin Obstet Gynecol. 2002;45:393-404.

5. Gupta SD, Khanna A, Gupta R. Maternal mortality ratio and predictors of maternal deaths in selected desert districts in Rajasthan: a community-based survey and case control study. Womens Health Issues. 2010;20:80-5.

6. Kongnyuy EJ, Mlava G, van den Broek N. Facilitybased maternal death review in three districts in the central region of Malawi: an analysis of causes and characteristics of maternal deaths. Womens Health Issues. 2009;19:14-20.

7. Bouvier-Colle MH, Mohangoo AD, Gissler M, Novak-Antolic Z, Vutuc C, Szamotulska K, et al. What about the mothers? An analysis of maternal mortality and morbidity in perinatal health surveillance systems in Europe. BJOG. 2012;119:880-90.

8. Surgers L, Valin N, Carbonne B, Bingen E, Lalande $\mathrm{V}$, Pacanowski J, et al. Evolving microbiological epidemiology and high fetal mortality in 135 cases of bacteremia during pregnancy and postpartum. Eur $\mathbf{J}$ Clin Microbiol Infect Dis. 2013;32:107-13.

9. Acosta CD, Bhattacharya S, Tuffnell D, Kurinczuk JJ, Knight M. Maternal sepsis: a Scottish populationbased case-control study. BJOG. 2012;119:474-83.

10. Kankuri E, Kurki T, Carlson P, Hiilesmaa V. Incidence, treatment and outcome of peripartum sepsis. Acta Obstet Gynecol Scand. 2003;82:730-5.

Cite this article as: Kumari A, Suri J, Mittal P. Descriptive audit of maternal sepsis in a tertiary care centre of North India. Int J Reprod Contracept Obstet Gynecol 2018;7:124-7. 